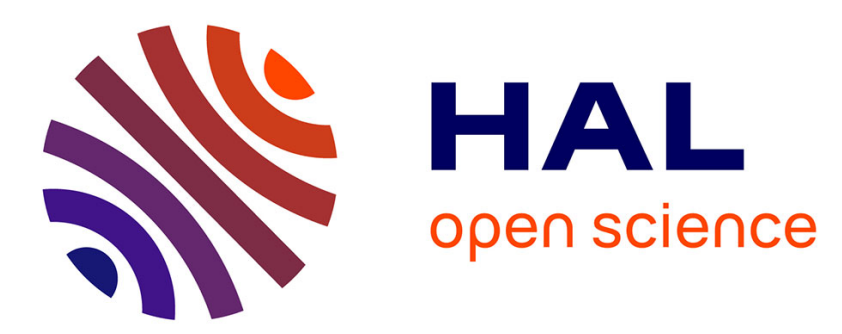

\title{
Control of the spin to charge conversion using the inverse Rashba-Edelstein effect
}

Soraya Sangiao, J.M. de Teresa, L. Morellon, I. Lucas, M.C. Martinez-Velarte, Michel Viret

\section{- To cite this version:}

Soraya Sangiao, J.M. de Teresa, L. Morellon, I. Lucas, M.C. Martinez-Velarte, et al.. Control of the spin to charge conversion using the inverse Rashba-Edelstein effect. Applied Physics Letters, 2015, 106, pp.172403. 10.1063/1.4919129 . cea-01498288

\section{HAL Id: cea-01498288 https://hal-cea.archives-ouvertes.fr/cea-01498288}

Submitted on 29 Mar 2017

HAL is a multi-disciplinary open access archive for the deposit and dissemination of scientific research documents, whether they are published or not. The documents may come from teaching and research institutions in France or abroad, or from public or private research centers.
L'archive ouverte pluridisciplinaire HAL, est destinée au dépôt et à la diffusion de documents scientifiques de niveau recherche, publiés ou non, émanant des établissements d'enseignement et de recherche français ou étrangers, des laboratoires publics ou privés. 


\title{
Control of the spin to charge conversion using the inverse Rashba-Edelstein effect
}

\author{
S. Sangiao, ${ }^{1,2,3}$ J. M. De Teresa, ${ }^{2,4}$ L. Morellon, ${ }^{2}$ I. Lucas, ${ }^{2,3}$ M. C. Martinez-Velarte, ${ }^{2}$ \\ and M. Viret ${ }^{1, a)}$ \\ ${ }^{1}$ Service de Physique de l'Etat Condensé, CEA Saclay, DSM/IRAMIS/SPEC, bat 772, CNRS UMR 3680, \\ F-91191 Gif-sur-Yvette, France \\ ${ }^{2}$ Laboratorio de Microscopías Avanzadas (LMA), Instituto de Nanociencia de Aragón (INA) and \\ Departamento de Física de la Materia Condensada, Universidad de Zaragoza, 50018 Zaragoza, Spain \\ ${ }^{3}$ Fundación ARAID, 50018 Zaragoza, Spain \\ ${ }^{4}$ Instituto de Ciencia de Materiales de Aragón (ICMA), Universidad de Zaragoza-CSIC, Facultad de Ciencias, \\ 50009 Zaragoza, Spain
}

(Received 3 February 2015; accepted 6 April 2015; published online 29 April 2015)

We show here that using spin orbit coupling interactions at a metallic interface it is possible to control the sign of the spin to charge conversion in a spin pumping experiment. Using the intrinsic symmetry of the "Inverse Rashba Edelstein Effect" (IREE) in a Bi/Ag interface, the charge current changes sign when reversing the order of the Ag and Bi stacking. This confirms the IREE nature of the conversion of spin into charge in these interfaces and opens the way to tailoring the spin sensing voltage by an appropriate trilayer sequence. (C) 2015 AIP Publishing LLC.

[http://dx.doi.org/10.1063/1.4919129]

Classical spintronics relies on the generation and manipulation of spin polarized electrical currents in magnetic conductors. It has been recently understood that spin currents can also be generated in non-magnetic materials using the spin-orbit coupling (SOC) interaction opening a new field called spin-orbitronics. The best known of these is the Spin Hall Effect (SHE) first introduced in the seventies. ${ }^{1,2}$ It relies on a preferential directional scattering of electrons of different spins by SOC on crystalline imperfections. It thus results in the generation of a transverse spin current when a charge current flows in a large SOC material like, for instance, Pt. The inverse SHE (ISHE) has been widely used lately to sense a spin current as the SOC interaction converts it into a (transverse) charge current. Very recently, another SOC effect based on the Rashba interaction has been evidenced. It stems from the joint action of the SOC and built-in electric potentials in two-dimensional electron gases (2DEGs) existing at surfaces, interfaces, or semiconductor quantum wells. In these systems, a charge current generates a non-zero spin density and transverse spin accumulation ${ }^{3,4}$ in virtue of the Rashba Hamiltonian

$$
H_{R}=\alpha_{R}(\vec{k} \times \vec{z}) \cdot \vec{s}
$$

where $\mathrm{s}$ is the spin vector, $\mathrm{k}$ is the momentum of charge carriers, $\mathrm{z}$ is the coordinate normal to the interface, and $\alpha_{R}$ is the Rashba coefficient, proportional to the induced internal electric field. When in contact with a magnetic material, this spin density can apply a torque on the magnetization, a mechanism put forward to explain experimental results on current induced domain wall motion and magnetization switching. ${ }^{5,6}$ Recently, a related effect, the Inverse Rashba Edelstein Effect (IREE) has been demonstrated ${ }^{7}$ where a spin current generated by ferromagnetic resonance $(\mathrm{FMR})^{8}$ in a magnetic

a)michel.viret@cea.fr layer, is converted into a charge current when flowing through an adjacent $\mathrm{Ag} / \mathrm{Bi}$ interface. $\mathrm{Bi}(111)$ is indeed known to produce interface states with many materials where strong Rashba coupling takes place ${ }^{9,10}$ and the Bi/Ag interface is known to be a particularly good one. ${ }^{7}$ Interestingly, because the asymmetrical potential at the interface is responsible for generating the transverse spin/charge density, one can envision to tailor the spin to charge conversion by shaping the asymmetric well. In particular, and quite straightforwardly, the charge current direction should be reversed when $\mathrm{Ag} / \mathrm{Bi}$ is replaced by $\mathrm{Bi} / \mathrm{Ag}$, i.e., the interface asymmetry is reversed. This is the aim of the present paper.

Controlling the stack sequence is not such a straightforward matter, mainly because of the tricky deposition of good quality Bi. Our trilayer system is therefore a little different, as shown in Figure 1. Spins are injected using the ferromagnetic resonance induced pumping from a 10-nm thick epitaxial Fe layer DC sputtered on a single-crystal $\mathrm{MgO}(001)$

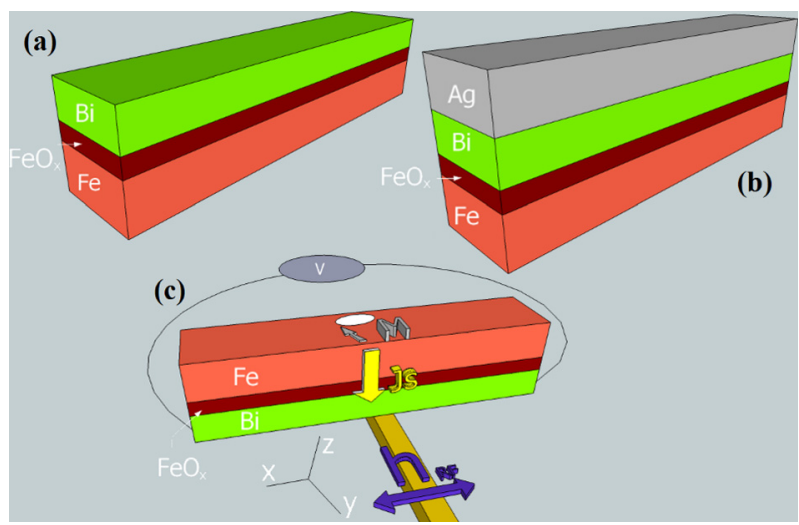

FIG. 1. Schematics of the Fe/Bi samples for ISHE measurements (a) and the $\mathrm{Fe} / \mathrm{Bi} / \mathrm{Ag}$ samples for IREE measurements (b). (c) Sketch of the experimental configuration. The dc field is along $y$ and the rf field along $x$. The spin current is injected into the $\mathrm{Bi}$ layer through the Fe-oxide layer. 
substrate. The films were grown at a temperature of $200^{\circ} \mathrm{C}$ in a combined pulsed laser deposition (PLD)-sputtering system with a base pressure of $<5 \times 10^{-9}$ Torr. They were then taken to air and introduced into another electron-beam evaporation system where bismuth layers with thickness between 7 and $40 \mathrm{~nm}$ were grown at room temperature in high vacuum $\left(10^{-7} \mathrm{Torr}\right)$. Importantly, an approximately 2 -nm thick native $\mathrm{Fe}$ oxide layer is formed after the air exposure. The $\mathrm{MgO}(001) / / \mathrm{Fe}(10 \mathrm{~nm}) / \mathrm{FeO}_{X}(\approx 2 \mathrm{~nm}) / \mathrm{Bi}(\mathrm{t})$ stacks were fully characterized by $\mathrm{X}$-ray diffraction and reflectivity and vibrating sample magnetometry.

For the measurements, we proceed as in Ref. 7 where the signals from $\mathrm{SiO}_{2} / / \mathrm{Bi} / \mathrm{Ag} / \mathrm{NiFe}$ systems were compared to the reference $\mathrm{SiO}_{2} / / \mathrm{Bi} / \mathrm{NiFe}$ bilayer. In this geometry (the spin current is coming from the top NiFe layer), the pure ISHE in $\mathrm{Bi}$ has the same sign as the IREE at the $\mathrm{Ag} / \mathrm{Bi}$ interface. ${ }^{7}$ In our trilayers (Fig. 1(b)), the spin current is generated from the bottom Fe layer and same comparison is made with the pure ISHE in the Bi of the simple system of Fig. 1(a). In our experiment, an rf field is generated parallel to the long axis of the sample by a $500-\mu \mathrm{m}$ wide Au microwave line as illustrated in Figure 1(c). The ISHE/IREE voltage is measured through two gold contacts separated by $7 \mathrm{~mm}$ by a lock-in technique at room temperature. The rf signal, modulated by a square wave at a few $\mathrm{kHz}$, is sent to the transmission line at frequencies between 8 and $14 \mathrm{GHz}$ and at 15 $\mathrm{dBm}$. The transmission cell was positioned between the poles of a rotating electromagnet providing dc magnetic fields up to $1.2 \mathrm{~T}$ at any in-plane angle.

When the static magnetic field and the microwave frequency fulfil the FMR condition, the precessing magnetization in the Fe layer generates a vertical spin current via spin pumping which is injected into the Bi layer through the native $\mathrm{Fe}$ oxide layer. It is then converted into a charge current in the Bi layer perpendicular to the magnetization direction by ISHE $^{11}$

$$
J_{C}=\frac{2 e}{\hbar} \Theta_{S H} J_{S}
$$

where e is the electron charge, $\hbar$ is the reduced Planck constant, $\mathrm{J}_{S}$ is the pumped spin current, and $\Theta_{S H}$ is the spin Hall angle which quantifies the conversion efficiency between spin and charge currents. This dc current flows along the $\mathrm{x}$ axis of Figure 1(c) and gives rise, in an open circuit configuration, to a dc voltage, $\mathrm{V}_{I S H}$.

The frequency dependence of this ISH voltage measured along the hard axis of the Fe film (Fe[110], y-direction in Figure 1(c)) is shown in Figure 2(a) for an $\mathrm{Fe}(10 \mathrm{~nm}) / \mathrm{Bi}(\mathrm{t})$ with $\mathrm{t}=7.7 \mathrm{~nm}$. As expected for ferromagnetic systems with a large magnetocrystalline anisotropy, two resonances can be observed along the hard direction at frequencies below a threshold value of the order of $10 \mathrm{GHz}$. The higher field ISH voltage peak corresponds to resonance in the saturated state where the magnetization and the static magnetic field are parallel, whereas these are not aligned for the lower field peak. The full dependence of the frequency on the static resonant magnetic field along the easy ( $\mathrm{Fe}[100]$, in-plane direction at $45^{\circ}$ from both the $\mathrm{x}$ and $\mathrm{y}$ axes) and hard axes is summarized in Figure 2(b). The obtained dispersion relation

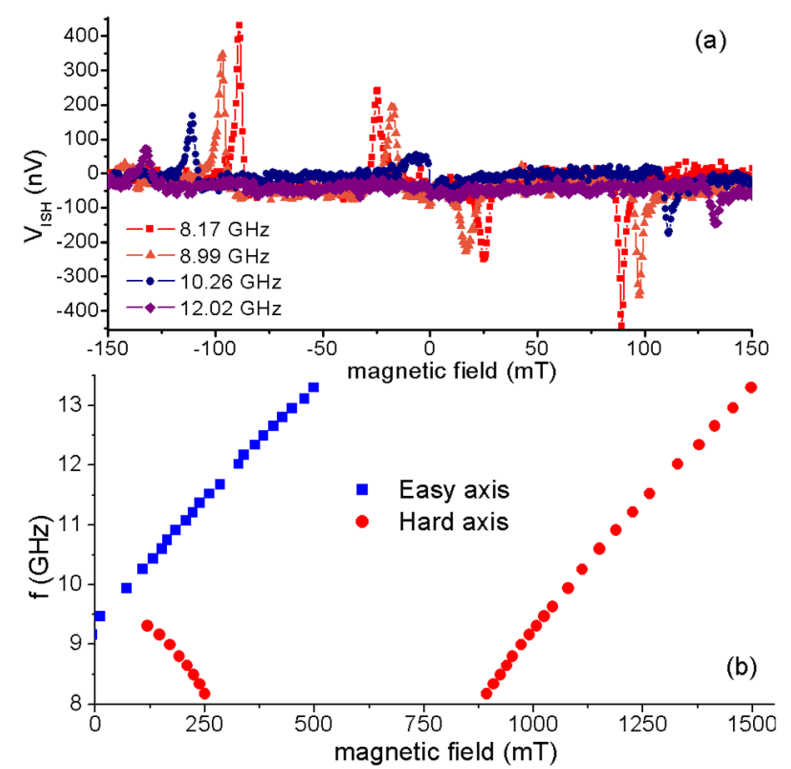

FIG. 2. (a) Frequency dependence of the ISH voltage measured for an $\mathrm{Fe}(10 \mathrm{~nm}) / \mathrm{Bi}(7.7 \mathrm{~nm})$ stack along the hard axis of the Fe film (y-direction in Figure 1(c)). The microwave power is $15 \mathrm{dBm}$. (b) Frequency dependence of the resonant magnetic field (dispersion relation) for the epitaxial Fe film along the easy and the hard axes.

of epitaxial Fe films is in very good agreement with the one previously determined by ISH measurements in $\mathrm{Fe} / \mathrm{Pt}$ stacks. $^{12}$

The estimation of the spin Hall angle in bismuth can be extracted from the Bi thickness dependence of the ISH voltage in the stacks $\mathrm{Fe}(10 \mathrm{~nm}) / \mathrm{FeO}_{X}(\approx 2 \mathrm{~nm}) / \mathrm{Bi}(\mathrm{t})$. This is plotted in Figure 3(a) where ISH voltages were measured at $9.46 \mathrm{GHz}$ and $15 \mathrm{dBm}$ for Bi thickness between 7 and $24 \mathrm{~nm}$. The signal increases from 270 to $360 \mathrm{nV}$ as the Bi thickness goes from 7.7 to $9.3 \mathrm{~nm}$ where it approaches its maximum and then decreases to $264 \mathrm{nV}$ as the thickness is increased to $22.5 \mathrm{~nm}$. This dependence with the thickness of the nonmagnetic layer is in good agreement with that recently reported by Emoto et al. ${ }^{13}$ in Py/Bi bilayers and by other authors in similar magnetic/non-magnetic bilayers. ${ }^{14}$ Interestingly, we do not observe the anomalous dependence reported by Hou et al. ${ }^{15}$ in Py/Bi bilayers. This effect was attributed to a modification of the spin Hall angle and the spin diffusion length $(0.019$ and $50 \mathrm{~nm}$, respectively, in the bulk) at the $\mathrm{Bi} / \mathrm{Py}$ interface $(-0.071$ and $2.4 \mathrm{~nm}$, respectively), due to the electric field across this interface which locally shifts the Fermi level in Bi. In our stacks, the presence of the native $\mathrm{Fe}$ oxide impedes the band bending at the magnetic layer/Bi contact and suppresses any anomalous contribution to the ISH voltage from interfacial effects.

The voltage due to the ISHE generated in our samples can be calculated using the following expression: ${ }^{16}$

$$
V_{I S H}=\frac{\Theta_{S H} e L P f g_{\uparrow \downarrow} \lambda_{s d} \sin ^{2} \theta}{\sigma_{F e} t_{F e}+\sigma_{B i} t_{B i}} \tanh \left(\frac{t_{B i}}{2 \lambda_{s d}}\right),
$$

where $\mathrm{f}$ is the frequency of the microwave field, $\sigma_{B i}$ and $\sigma_{F e}$ are the charge conductivities of $\mathrm{Bi}$ and $\mathrm{Fe}$ layers and $\mathrm{t}_{B i}$ and $\mathrm{t}_{\mathrm{Fe}}$ are their thicknesses, $\mathrm{L}$ is the length of the $\mathrm{Fe} / \mathrm{Bi}$ stack being excited by the microwave field $(500 \mu \mathrm{m}), \mathrm{g}_{\uparrow \downarrow}$ is the spin-mixing conductance at the $\mathrm{Fe} / \mathrm{Bi}$ interface, $\lambda_{s d}$ is the 

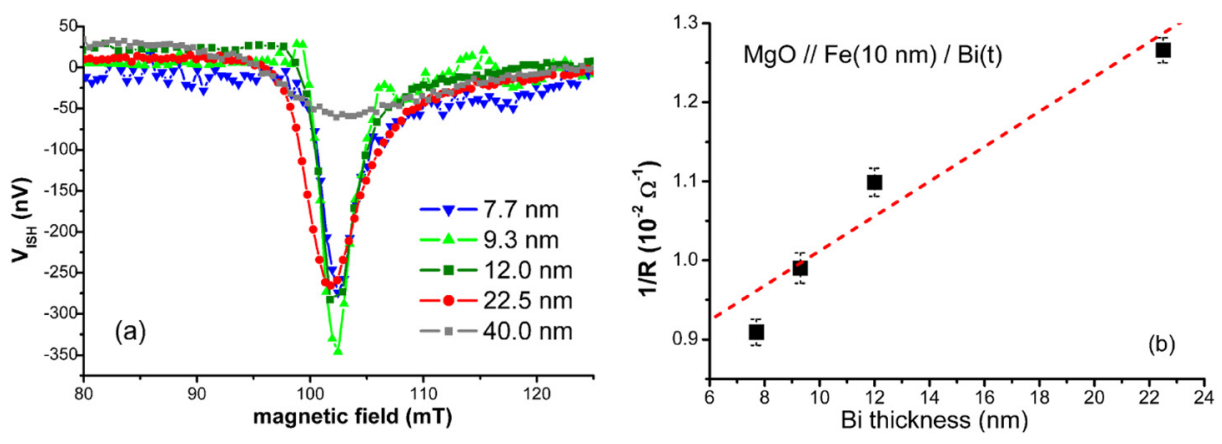

FIG. 3. (a) ISH voltage measured in $\mathrm{Fe}(10 \mathrm{~nm}) / \mathrm{Bi}$ stacks with different $\mathrm{Bi}$ thicknesses. Microwave frequency and power are $9.46 \mathrm{GHz}$ and $15 \mathrm{dBm}$, respectively. (b) Bi thickness dependence of the conductance of the $\mathrm{Fe} / \mathrm{Bi}$ stacks where the dotted line is a linear fit. (c) Dependence of ISH voltage on Bi thickness for a microwave frequency of $8.97 \mathrm{GHz}$. The line is a fit to Eq. (3) for $\Theta_{S H}=0.016$ and $\lambda_{s d}=16 \mathrm{~nm}$. (d) Variation of the spin hall angle and the spin-diffusion length derived from the fits of the Bi-thickness dependence of the ISH voltage at different microwave frequencies.
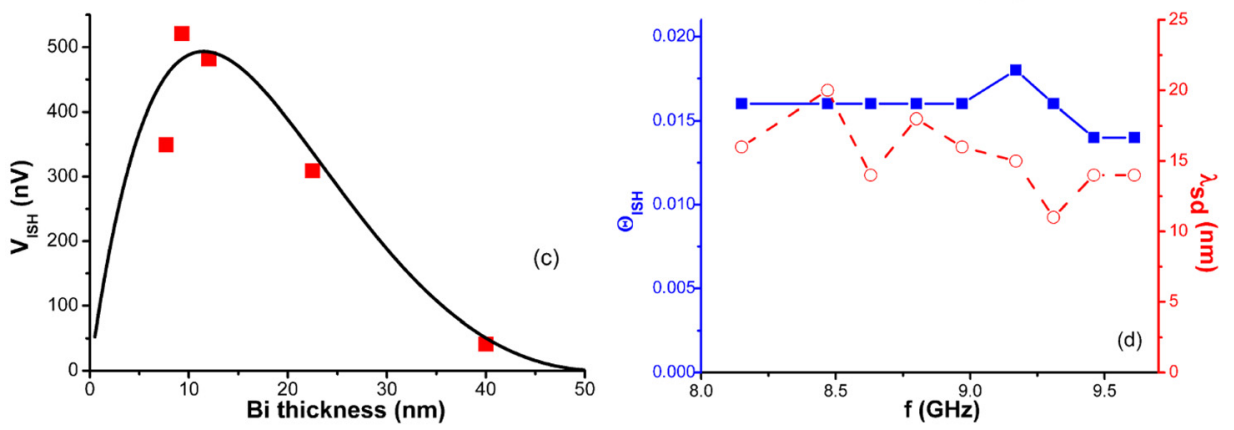

spin-diffusion length in $\mathrm{Bi}, \theta$ is the angle of magnetization precession in the $\mathrm{Fe}$ layer, and $\mathrm{P}$ is the correction factor accounting for the elliptical nature of the magnetization trajectory due to the strong perpendicular demagnetization fields. ${ }^{17}$ Moreover, the difference in the Gilbert damping parameter, $\alpha$, for a $\mathrm{Fe} / \mathrm{Bi}$ bilayer with a sufficiently thick $\mathrm{Bi}$ layer, $\alpha_{F e / B i}$, and for a Fe sample without Bi capping, $\alpha_{F e}$, allows the determination of the spin-mixing conductance ${ }^{18}$

$$
g_{\uparrow \downarrow}=\frac{4 \pi M_{S} t_{F e}}{g \mu_{B}}\left(\alpha_{F e / B i}-\alpha_{F e}\right),
$$

where $\mathrm{g}$ is the Landé factor, $\mu_{B}$ is the Bohr magneton, and $\mathrm{M}_{S}$ is the Fe saturation magnetization. The values of the Gilbert damping parameter obtained for the reference sample $\left(\mathrm{t}_{B i}=0\right)$ and for one of the thickest stacks studied $\left(\mathrm{t}_{B i}=22.5 \mathrm{~nm}\right)$ are $\alpha_{F e}=0.0073$ and $\alpha_{F e / B i}=0.0084$, respectively. These values, together with the parameters extracted from the fitting of the in-plane dispersion relation data in $\mathrm{Fe}$ using the Kittel equation, ${ }^{19} \mathrm{M}_{S}=1720 \mathrm{emu} \mathrm{cm}^{-3}$ and $\mathrm{H}_{a}=550 \mathrm{Oe}$, allow us to estimate the spin-mixing conductance at the interface $\mathrm{Fe} / / \mathrm{Bi}$ : $\mathrm{g}_{\uparrow \downarrow}=1.02 \times 10^{18} \mathrm{~m}^{-2}$ which is significantly lower than the one reported previously for the $\mathrm{Py} / \mathrm{Bi}$ interface $\left(1.06 \times 10^{19} \mathrm{~m}^{-2}\right)$. We attribute this to the insulating nature of the Fe oxide layer present at the interface, which partly suppresses the spin pumping from the ferromagnetic layer. Hence, at the price of this reduced transparency, interfacial contributions are overcome and only "bulk" ISHE in Bi can be considered. Moreover, the analysis of the dc voltage dependence on the Bi thickness (see Fig. 3(c)) demonstrates the predominance of bulk contributions to the dc signal measured in our Fe/Bi stacks.

The variation of the $\mathrm{Fe} / \mathrm{Bi}$ bilayers conductance with $\mathrm{Bi}$ thickness is displayed in Figure 3(b). The resulting linear variation indicates a constant resistivity of the $\mathrm{Bi}$ layer: $\rho_{B i}=132 \mu \Omega \mathrm{cm}$, in very good agreement with the bulk value $(130 \mu \Omega \mathrm{cm})$. Fitting the Bi thickness dependence of the ISH voltage at different microwave frequencies with the expression given by Eq. (2) (see Fig. 3(c)), we have extracted the value of the spin Hall angle and the spin diffusion length at each frequency in Fig. 3(d). They are both found to be, within the overall experimental error, frequency independent, which is consistent with the fact that $\Theta_{S H}$ and $\lambda_{s d}$ are material specific parameters. Taking into account all the parameters' values determined at different frequencies, we obtain $\Theta_{S H}=0.0158(8)$ and $\lambda_{s d}=15.3(5) \mathrm{nm}$. This value for the spin Hall angle is in reasonably good agreement with the ones previously reported (0.019(2) in Ref. 15 and 0.02 in Ref. 13). Furthermore, the spin diffusion length we obtain is also in between the two previously reported values estimated from ISH voltage measurements (50(12) $\mathrm{nm}$ in Ref. 15 and $8 \mathrm{~nm}$ in Ref. 13). This spread in $\lambda_{s d}$ can be attributed to the different crystalline qualities of the $\mathrm{Bi}$ layers under study: ours are polycrystalline but strongly textured, with the trigonal axis ([001] in hexagonal indexing) perpendicular to the plane of the film; ${ }^{20}$ whereas, the Bi layers in Ref. 13 are amorphous and those in Ref. 15 are polycrystalline with no preferred orientation.

After analysing the spin-to-charge conversion due to the pure ISHE in $\mathrm{Bi}$, an $\mathrm{Ag}$ layer is added on top in order to study the effect of the $\mathrm{Bi} / \mathrm{Ag}$ interface. For this purpose, we have grown an $\mathrm{Fe}(10.0 \mathrm{~nm}) / \mathrm{Bi}(8.0 \mathrm{~nm}) / \mathrm{Ag}(20.0 \mathrm{~nm})$ stack, represented in Figure 1(b), in which the Fe layer is again natively oxidised and the $\mathrm{Bi} / \mathrm{Ag}$ bilayer is deposited in situ using the same evaporation conditions. Similar to the Fe/Bi stacks, a vertical dc spin current $\mathrm{J}_{S}$ is pumped from the $\mathrm{Fe}$ layer set in resonance. Inside the Bi layer, this spin current decays exponentially on a length scale given by $\lambda_{s d}$, leading to a spin current impinging the $\mathrm{Bi} / \mathrm{Ag}$ interface proportional to $\exp \left(-\mathrm{t}_{B i} / \lambda_{s d}\right)$. For our $\operatorname{Fe}(10.0 \mathrm{~nm}) / \mathrm{Bi}(8.0 \mathrm{~nm}) /$ $\mathrm{Ag}(20.0 \mathrm{~nm})$ stack, this corresponds to a decrease of 0.59 with respect to the spin current injected into the Bi layer. The large Rashba coupling at the $\mathrm{Bi} / \mathrm{Ag}$ interface converts this spin current into a charge current $\left(\mathrm{I}_{\text {IREE }}\right)$ carried by the Rashba 2DEG. ${ }^{3,21}$ This gives rise, in an open circuit configuration, to the IREE dc voltage, $\mathrm{V}_{\text {IREE }}$.

In Figure 4, we compare the field dependence of the dc voltage measured in two different stacks: $\mathrm{Fe}(10.0 \mathrm{~nm}) /$ 


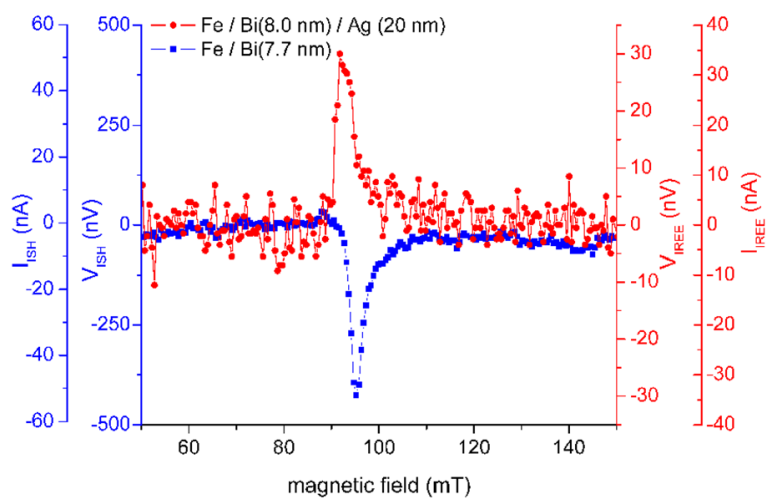

FIG. 4. Measured DC voltage and inferred DC, for two stacks: Fe/ $\mathrm{Bi}(7.7 \mathrm{~nm})$ (blue squares, red axes) and $\mathrm{Fe} / \mathrm{Bi}(8.0 \mathrm{~nm}) / \mathrm{Ag}(20 \mathrm{~nm})$ (red dots, right axes) at positive magnetic fields. Microwave frequency and power are, respectively, $8.80 \mathrm{GHz}$ and $15 \mathrm{dBm}$.

$\mathrm{Bi}(7.7 \mathrm{~nm})$ and the $\mathrm{Fe}(10.0 \mathrm{~nm}) / \mathrm{Bi}(8.0 \mathrm{~nm}) / \mathrm{Ag}(20.0 \mathrm{~nm})$ with the added $\mathrm{V}_{\text {IREE }}$ contribution. The first important point is the sign reversal between (negative) $\mathrm{Fe} / \mathrm{Bi}$ and (positive) $\mathrm{Fe} / \mathrm{Bi} / \mathrm{Ag}$. In their recent study, Rojas-Sanchez et al. ${ }^{7}$ obtained the same sign in both $\mathrm{NiFe} / \mathrm{Bi}$ and $\mathrm{NiFe} / \mathrm{Ag} / \mathrm{Bi}$ stacks demonstrating that the IREE in $\mathrm{Ag} / \mathrm{Bi}$ is in the same direction as the pure ISHE in Bi. Therefore, our measurement of an opposite dc voltage in $\mathrm{Fe} / \mathrm{Bi}$ and $\mathrm{Fe} / \mathrm{Bi} / \mathrm{Ag}$ indicates that the sign of $\mathrm{V}_{\text {IREE }}$ in $\mathrm{Fe} / \mathrm{Bi} / \mathrm{Ag}$ is opposite to that in $\mathrm{NiFe} / \mathrm{Ag} / \mathrm{Bi}$. This implies that the Rashba charge current, $\mathrm{I}_{\text {IREE }}$, is indeed reversed through the inversion of the order of the $\mathrm{Ag}$ and $\mathrm{Bi}$ layers composing the interface. Although the measured ISH voltage in $\mathrm{Fe} / \mathrm{Bi}$ is larger than the IREE voltage in $\mathrm{Fe} / \mathrm{Bi} / \mathrm{Ag}$, the values converted into charge currents are comparable (see outer axes in Figure 4) despite the fact that the spin current injected at the $\mathrm{Bi} / \mathrm{Ag}$ interface is lower than that at the Bi layer, as explained above. The larger dc charge current obtained in the $\mathrm{Fe} / \mathrm{Bi} / \mathrm{Ag}$ stack cannot be explained via the contribution from ISHE in Ag due to the small and, more importantly, positive, spin Hall angle in $\mathrm{Ag}$, $\Theta_{S H}(\mathrm{Ag})=0.0068(7){ }^{22}$ Thus from the inferred IREE current in $\mathrm{Fe} / \mathrm{Bi} / \mathrm{Ag}$, we can extract the value of the IREE length, $\lambda_{\text {IREE }}$, characterizing the conversion of the injected spin current into a $2 \mathrm{D}$ charge current ${ }^{7}$

$$
J_{C}=\lambda_{I R E E} J_{S},
$$

where $\mathrm{J}_{C}$ is the $2 \mathrm{D}$ charge current density in $\mathrm{A} \mathrm{m}^{-1}$ and $\mathrm{J}_{S}$ is the spin current density injected at the interface in $\mathrm{A} \mathrm{m}^{-2}$. From our measurements, we estimate the charge current density to be $\mathrm{J}_{C}=1.75 \times 10^{-5} \mathrm{~A} \mathrm{~m}^{-1}$ and the spin current density reaching the interface to be $\mathrm{J}_{S}=-5.23 \times 10^{4} \mathrm{~A} \mathrm{~m}^{-2}$. Thus, we obtain an IREE characteristic length $\lambda_{\text {IREE }}=-0.33 \mathrm{~nm}$. This value is in very good agreement with the previous measurement $^{7}$ of $\lambda_{\text {IREE }}=+0.2 \mathrm{~nm}$ for $\mathrm{NiFe}(15 \mathrm{~nm}) / \mathrm{Ag}(\mathrm{tAg}) /$ $\operatorname{Bi}(8 \mathrm{~nm})$ stacks with $\mathrm{t}_{A g}=5 \mathrm{~nm}$ and $\lambda_{\text {IREE }}=+0.33 \mathrm{~nm}$ for $\mathrm{t}_{A g}=20 \mathrm{~nm}$.

To summarize, we have determined here, from the ISHE measurements on $\mathrm{Fe} / \mathrm{Bi}$ stacks, values for the spin Hall angle and the spin diffusion length in $\mathrm{Bi}$ of $\Theta_{S H}=0.0158(8)$ and $\lambda_{s d}=15.3(5) \mathrm{nm}$, in good agreement with the previous reports. ${ }^{13,15}$ Based on these results, we have shown that the contribution due to IREE in $\mathrm{Fe} / \mathrm{Bi} / \mathrm{Ag}$ stacks has an opposite sign, thus demonstrating that the sense of the Rashba charge current is determined by the order of the $\mathrm{Ag}$ and $\mathrm{Bi}$ sequence. This confirms the IREE nature of the spin to charge conversion at the $\mathrm{Ag} / \mathrm{Bi}$ interface and paves the way for future research on design of spintronic devices, where the controlled generation and detection of spin currents are required.

We would like to acknowledge financial support from the European Commission through the Marie Curie Intra European Fellowship Project No. 301656: AtomicFMR, funded by the 7th Framework Programme. This work was supported by Spanish Ministry of Economy and Competitivity through Project No. MAT2011-27553-C02, including FEDER funds, and by the Aragon Regional Government. We would also like to acknowledge interesting discussions with Laurent Vila and Albert Fert and thank them for a critical reading of this manuscript.

${ }^{1}$ M. I. Dyakonov and V. I. Perel, JETP Lett. 13, 467 (1971).

${ }^{2}$ M. I. Dyakonov and V. I. Perel, Phys. Lett. A 35, 459 (1971).

${ }^{3}$ V. M. Edelstein, Solid State Commun. 73, 233 (1990).

${ }^{4}$ S. Bhattacharjee, S. Singh, D. Wang, M. Viret, and L. Bellaiche, J. Phys.: Condens. Matter 26, 315008 (2014).

${ }^{5}$ I. M. Miron, G. Gaudin, S. Auffret, B. Rodmacq, A. Schuhl, S. Pizzini, J. Vogel, and P. Gambardella, Nat. Mater. 9, 230 (2010).

${ }^{6}$ I. M. Miron, K. Garello, G. Gaudin, P. J. Zermatten, M. V. Costache, S. Auffret, S. Bandiera, B. Rodmacq, A. Schuhl, and P. Gambardella, Nature 476, 189 (2011).

${ }^{7}$ J. C. Rojas Sanchez, L. Vila, G. Desfonds, S. Gambardelli, J. P. Attané, J. M. De Teresa, C. Magen, and A. Fert, Nat. Commun. 4, 2944 (2013).

${ }^{8}$ Y. Tserkovnyak, A. Brataas, G. E. W. Bauer, and B. I. Halperin, Rev. Mod. Phys. 77, 1375 (2005).

${ }^{9}$ Yu. M. Koroteev, G. Bihlmayer, J. E. Gayone, E. V. Chulkov, S. Blugel, P. M. Echenique, and Ph. Hofmann, Phys. Rev. Lett. 93, 046403 (2004).

${ }^{10}$ C. R. Ast, J. Henk, A. Ernst, L. Moreschini, M. C. Falub, D. Pacile, P. Bruno, K. Kern, and M. Grioni, Phys. Rev. Lett. 98, 186807 (2007).

${ }^{11}$ E. Saitoh, M. Ueda, H. Miyajima, and G. Tatara, Appl. Phys. Lett. 88, 182509 (2006).

${ }^{12}$ E. Th. Papaioannou, P. Fuhrmann, M. B. Jungfleisch, T. Bracher, P. Pirro, V. Lauer, J. Losch, and B. Hillebrands, Appl. Phys. Lett. 103, 162401 (2013).

${ }^{13}$ H. Emoto, Y. Ando, E. Shikoh, Y. Fuseya, T. Shinjo, and M. Shiraishi, J. Appl. Phys. 115, 17C507 (2014).

${ }^{14}$ C. Hahn, G. de Loubens, O. Klein, M. Viret, V. V. Naletov, and J. Ben Youssef, Phys. Rev. B 87, 174417 (2013).

${ }^{15}$ D. Hou, Z. Qiu, K. Harii, Y. Kajiwara, K. Uchida, Y. Fujikawa, H. Nakayama, T. Yoshino, T. An, K. Ando, X. Jin, and E. Saitoh, Appl. Phys. Lett. 101, 042403 (2012).

${ }^{16}$ O. Mosendz, V. Vlaminck, J. E. Pearson, F. Y. Fradin, G. E. W. Bauer, S. D. Bader, and A. Hoffmann, Phys. Rev. B 82, 214403 (2010).

${ }^{17}$ K. Ando, T. Yoshino, and E. Saitoh, Appl. Phys. Lett. 94, 152509 (2009).

${ }^{18}$ R. Urban, G. Woltersdorf, and B. Heinrich, Phys. Rev. Lett. 87, 217204 (2001).

${ }^{19}$ C. Kittel, Introduction to Solid State Physics, 7th ed. (Wiley, 1996).

${ }^{20}$ N. Marcano, S. Sangiao, C. Magen, L. Morellon, M. R. Ibarra, M. Plaza, L. Perez, and J. M. De Teresa, Phys. Rev. B 82, 125326 (2010).

${ }^{21}$ Y. A. Bychkov and E. I. Rashba, JETP Lett. 39, 78 (1984).

${ }^{22}$ H. L. Wang, C. H. Du, Y. Pu, R. Adur, P. C. Hammel, and F. Y. Yang, Phys. Rev. Lett. 112, 197201 (2014). 